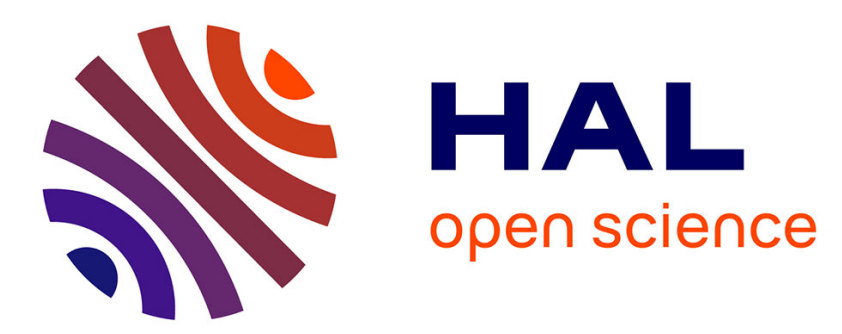

\title{
A Bayesian method to estimate the depth and the range of phonating sperm whales using a single hydrophone
}

Christophe Laplanche

\section{To cite this version:}

Christophe Laplanche. A Bayesian method to estimate the depth and the range of phonating sperm whales using a single hydrophone. Journal of the Acoustical Society of America, 2007, Vol. 121, pp. 1519-1528. 10.1121/1.2436644 . hal-00797709

\section{HAL Id: hal-00797709 \\ https://hal.science/hal-00797709}

Submitted on 7 Mar 2013

HAL is a multi-disciplinary open access archive for the deposit and dissemination of scientific research documents, whether they are published or not. The documents may come from teaching and research institutions in France or abroad, or from public or private research centers.
L'archive ouverte pluridisciplinaire HAL, est destinée au dépôt et à la diffusion de documents scientifiques de niveau recherche, publiés ou non, émanant des établissements d'enseignement et de recherche français ou étrangers, des laboratoires publics ou privés. 


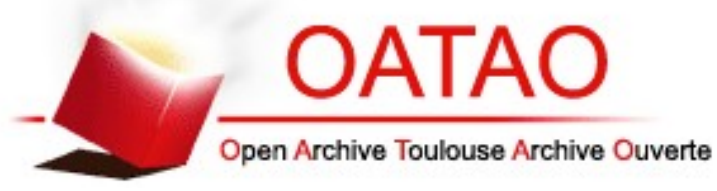

\section{Open Archive Toulouse Archive Ouverte (OATAO)}

OATAO is an open access repository that collects the work of Toulouse researchers and makes it freely available over the web where possible.

This is an author-deposited version published in: http://oatao.univ-toulouse.fr/ Eprints ID: 5607

To link to this article: DOI: $10.1121 / 1.2436644$

http://dx.doi.org/10.1121/1.2436644

To cite this version: Laplanche, Christophe A Bayesian method to estimate the depth and the range of phonating sperm whales using a single hydrophone. (2007) The Journal of the Acoustical Society of America (JASA), 9 ol. 121 ( $\mathrm{n}^{\circ}[3)$. pp. 1519-1528. ISSN 0001-4966

Any correspondence concerning this service should be sent to the repository administrator: staff-oatao@inp-toulouse.fr 


\title{
A Bayesian method to estimate the depth and the range of phonating sperm whales using a single hydrophone
}

\author{
Christophe Laplanche ${ }^{\text {a) }}$ \\ Laboratoire Images, Signaux et Systèmes Intelligents, Groupe Ingénierie des Signaux Neuro-Sensoriels, \\ Université Paris 12, Créteil, France
}

\begin{abstract}
Some bioacousticians have used a single hydrophone to calculate the depth/range of phonating diving animals. The standard one-hydrophone localization method uses multipath transmissions (direct path, sea surface, and seafloor reflections) of the animal phonations as a substitute for a vertical hydrophone array. The standard method requires three multipath transmissions per phonation. Bioacousticians who study foraging sperm whales usually do not have the required amount of multipath transmissions. However, they usually detect accurately (using shallow hydrophones towed by research vessels) direct path transmissions and sea surface reflections of sperm whale phonations (clicks). Sperm whales emit a few thousand clicks per foraging dive, therefore researchers have this number of direct path transmissions and this number of sea surface reflections per dive. The author describes a Bayesian method to combine the information contained in those acoustic data plus visual observations. The author's tests using synthetic data show that the accurate estimation of the depth/range of sperm whales is possible using a single hydrophone and without using any seafloor reflections. This method could be used to study the behavior of sperm whales using a single hydrophone in any location no matter what the depth, the relief, or the constitution of the seafloor might be.
\end{abstract}

[DOI: $10.1121 / 1.2436644]$

\section{INTRODUCTION}

Sperm whales undertake long foraging dives to catch their prey. They breathe at the sea surface, fluke-up and swim downwards to reach their prey, hunt at depth, and reascend back to the sea surface (Miller et al., 2004a). During foraging dives, sperm whales emit echolocation clicks (Backus and Schevill, 1966). They emit echolocation clicks at a tremendous source level (Møhl et al., 2003, 2000) and in series (Whitehead and Weilgart, 1990).

Since sperm whales emit long series of clicks of high source level, passive acoustics is an effective tool to study the foraging behavior of these animals. Researchers have developed and used different passive acoustic localization techniques. These techniques require synchronous recordings made on tridimensional (Watkins and Schevill, 1972), bidimensional (Thode, 2004), or unidimensional (Villadsgaard et al., 2007) arrays of hydrophones.

To locate the sound source using an array of $n$ hydrophones, one would need to isolate one signal emitted by the source and to measure the $n$ times of arrival (TOA) of this signal on the $n$ hydrophones of the array. The differences between TOAs (TOAD) are calculated. A TOAD provides information on the location of the source: The source is on a sheet of a two-sheeted hyperboloid of geometry given by the TOAD itself and by the location of the hydrophones used to calculate the TOAD. By repeating this localization process using different TOADs, one can, using the required amount of hydrophones (Spiesberger, 2001), geometrically or ana-

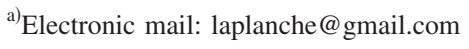

lytically compute the intersection of the hyperboloid sheets and therefore be able to more accurately identify the location of the source. A unidimensional array requires at least three hydrophones (Villadsgaard et al., 2007). In this case the hyperboloid sheets defined by the TOADs intersect into a circle. The plane containing this circle is perpendicular to the line of the array and the center of the circle sits on this line. Therefore, if the unidimensional array is vertical, then the circle is horizontal, and its depth and radius give the depth and the horizontal range of the sound source.

One noteworthy passive acoustic localization technique requires a single hydrophone [see for instance Thode et al. (2002) or Laplanche et al. (2005)]. Signals emitted by sound sources may reflect on the sea surface and the seafloor while propagating to the hydrophones. The detection on a hydrophone of the echoes from the surface/seafloor serves as a substitute of an unidimensional vertical hydrophone array (Urick, 1983). By measuring the TOADs of the echoes (later referred to as echo delays) relative to the nonreflected transmitted signal, one can calculate the depth and the range of the sound source. If the source is a phonating sperm whale, one can theoretically, by repeating the localization process on every click emitted by the whale during a whole dive, plot the values of the depth and the range of the whale during this dive.

Unfortunately, sperm whales usually forage above continental slopes or abyssal plains, i.e., areas of either deep or high relief seafloor. One can seldom clearly detect the seafloor echoes of clicks emitted by sperm whale using a hydrophone (close to the sea surface) towed by a research vessel. Usually one can only detect seafloor echoes at the beginning 
of the whale's dive, while the whale both swims and clicks downwards (Thode et al., 2002). In low sea state conditions, using a hydrophone close to the sea surface, one can however clearly detect surface echoes during the whole dive [see for instance Thode (2004) or Laplanche et al. (2005)].

Nevertheless, the measurement of a single echo delay (e.g., a surface echo delay) is not enough to calculate the depth and the range of a phonating sperm whale, since, as aforementioned, by using a single TOAD one can only know that the whale is on a hyperboloid sheet. Is it not possible to more accurately identify the location of the whale using a single hydrophone but not using seafloor echoes?

Actually one can still estimate the depth and the range of the whale under such constraints, and the aim of this work is to demonstrate the feasibility of this process. Every single surface echo delay contains information regarding the location of the whale. By combining the pieces of information contained in the set of the surface echo delays of the clicks emitted by the whale during a dive, one should be able to give a more accurate description of the location of the whale during this dive. The efficient combining of these pieces of information can be achieved in a Bayesian frame. The Bayesian approach has already proven to be efficient to locate sound sources using TOADs (Spiesberger, 2005).

The author proposes a Bayesian passive acoustic technique to estimate the depth and the range of foraging sperm whales using a single hydrophone and without using any seafloor echoes. To be applied, this technique requires a set of values of the sea surface echo delay of clicks emitted by the whale during the whole dive. It also requires the measurement, by a visual observer, of the approximate location (i.e., range and azimuth relative to the research vessel) of the whale when beginning and ending the foraging dive.

\section{MATERIAL AND METHODS}

\section{A. Trajectory model}

First, one would need a representation of the underwater trajectory of the whale using a mathematical model. Let $t^{(A)}$ be the time when the sperm whale flukes-up and starts diving, $t^{(B)}$ the time when the whale starts clicking, $t^{(C)}$ the time when the whale stops clicking, and $t^{(D)}$ the time when the whale resurfaces. The author will decompose the trajectory of the whale for $t \in\left[t^{(B)}, t^{(C)}\right]$ into $n \in \mathbb{N}^{*}$ pieces of equal duration $\tau_{0}=\left(t^{(C)}-t^{(B)}\right) / n$ (Fig. 1). Let $t^{(s)}=t^{(B)}+(s-1) \tau_{0}$ be the time when the whale is at the beginning $(s \in\{1, \ldots, n\})$ or at the end $(s \in\{2, \ldots, n+1\})$ of such trajectory pieces.

Let $E^{(s)}$ be the location in the terrestrial reference frame, let $z^{(s)}$ be the depth, let $r^{(s)}$ be the horizontal range, and let $\theta^{(s)}$ be the azimuth of the whale at time $t^{(s)}$ (Fig. 2). Let $E_{p}^{(s)}$ and $H_{p}$ be the projections of $E^{(s)}$ and $H(H$ is the location of the hydrophone) on a horizontal plane. Let $\gamma_{b}^{(s)}$ and $\gamma_{e}^{(s)}$ be the angles $\left(E_{p}^{(s)} \vec{E}_{p}^{(s+1)}, H_{p} \vec{E}_{p}^{(s)}\right)$ and $\left(E_{p}^{(s)} \vec{E}_{p}^{(s+1)}, H_{p} \vec{E}_{p}^{(s+1)}\right)$.

The value of $n$ is chosen high enough to be able to assume that the whale moves at constant speed and constant heading for $t \in\left[t^{(s)}, t^{(s+1)}\right](s \in\{1, \ldots, n\})$. Let $\mathcal{S}^{(s)}$ $=\left[E^{(s)} E^{(s+1)}\right]$ be the segment defining the location of the whale for $t \in\left[t^{(s)}, t^{(s+1)}\right]$. Let $v_{z}^{(s)} \in \mathbb{R}$ and $v_{r}^{(s)} \in \mathbb{R}^{+}$be the vertical and horizontal speeds of the whale along the seg-

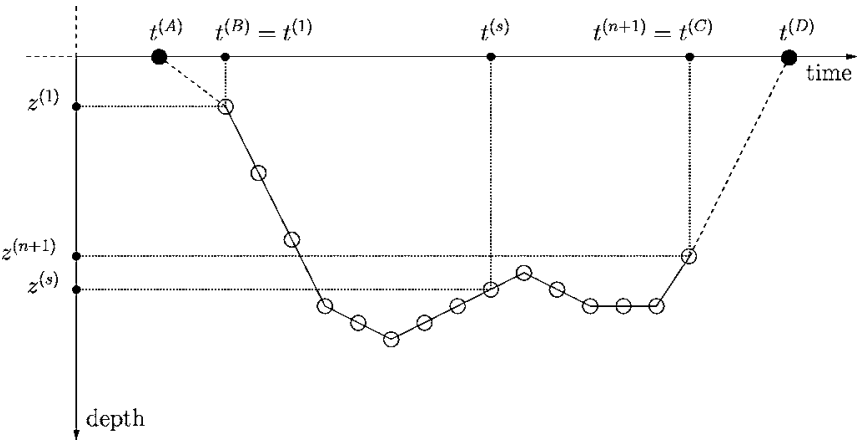

FIG. 1. The whale dives at $t=t^{(A)}$, starts clicking at $t=t^{(B)}$, stops clicking at $t=t^{(C)}$, and resurfaces at $t=t^{(D)}$. The whale is at the depth $z=z^{(s)}$ at $t=t^{(s)}$ $=t^{(B)}+(s-1) \tau_{0}(s \in\{1, \ldots, n+1\}$, in this example $n=14)$. The vertical speed of the whale is constant and equal to $v_{z}^{(s)}$ for $t \in\left[t^{(s)}, t^{(s+1)}\right]$.

ment $\mathcal{S}^{(s)}$. Each segment $\mathcal{S}^{(s)}$ is entirely defined by the coordinates of the points $E^{(s)}$ and $E^{(s+1)}$. The trajectory of the whale for $t \in\left[t^{(B)}, t^{(C)}\right]$ is labeled $\mathcal{T}$. It itself is entirely defined by the location of the summits $E^{(s)}(s \in\{1, \ldots, n+1\})$, that is to say $\mathcal{T} \equiv\left(E^{(1)}, \ldots, E^{(n+1)}\right)$. This definition of $\mathcal{T}$ requires, by using the coordinates in the terrestrial reference frame of the $n+1$ summits $E^{(s)}$, a set of $3 n+3$ parameters.

One can then define the trajectory $\mathcal{T}$ using the depth, range, and heading of the whale. Each segment $\mathcal{S}^{(s)}$ can be recursively defined by writing

$$
\begin{aligned}
& \mathcal{S}^{(1)} \equiv\left(z^{(1)}, r^{(1)}, \theta^{(1)}, z^{(2)}, r^{(2)}, \gamma_{b}^{(1)}\right), \\
& \mathcal{S}^{(s)} \equiv\left(z^{(s+1)}, r^{(s+1)}, \gamma_{b}^{(s)} \mid \mathcal{S}^{(s-1)}\right) \quad \text { for } s \in\{2, \ldots, n\}
\end{aligned}
$$

and the trajectory $\mathcal{T}$ is entirely defined by the set of $3 n+3$ parameters

$$
\mathcal{T} \equiv\left(z^{(1)}, r^{(1)}, \theta^{(1)}, z^{(2)}, r^{(2)}, \gamma_{b}^{(1)}, \ldots, z^{(n+1)}, r^{(n+1)}, \gamma_{b}^{(n)}\right) .
$$

This leads to the following definition of $\mathcal{T}$, which is required by the algorithm described later. Let $\Delta_{\gamma}^{(s)}=\gamma_{b}^{(s)}$ $-\gamma_{e}^{(s-1)}$ be the change of heading of the whale at time $t^{(s)}$. Each segment $\mathcal{S}^{(s)}$ is recursively defined by writing

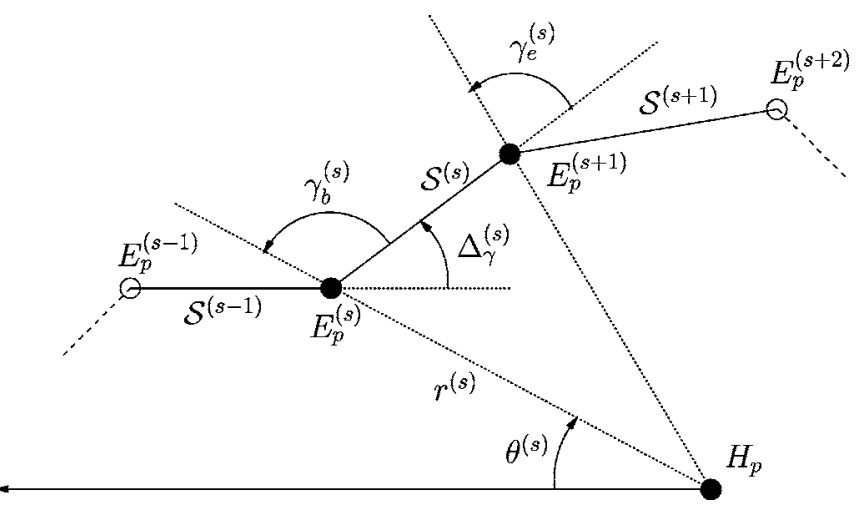

FIG. 2. The whale is at $E^{(s)}$ at $t=t^{(s)}$. The hydrophone is at $H . E_{p}^{(s)}$ and $H_{p}$ are the projections of $E^{(s)}$ and $H$ on a horizontal plane. The whale moves in a constant heading and at a constant horizontal speed $v_{r}^{(s)}$ for $t \in\left[t^{(s)}, t^{(s+1)}\right]$ along the segment $\mathcal{S}^{(s)}$ to reach $E^{(s+1)}$ at $t=t^{(s+1)}$. The angles $\gamma_{b}^{(s)}$ and $\gamma_{e}^{(s)}$ are defined as the angles between $\left(E_{p}^{(s)} E_{p}^{(s+1)}\right)$ and $\left(H_{p} E_{p}^{(s)}\right)$, and $\left(E_{p}^{(s)} E_{p}^{(s+1)}\right)$ and $\left(H_{p} E_{p}^{(s+1)}\right)$, respectively. The change of heading from $\mathcal{S}^{(s-1)}$ to $\mathcal{S}^{(s)}$ is $\Delta_{\gamma}^{(s)}$ $=\gamma_{e}^{(s-1)}-\gamma_{b}^{(s)}$. The horizontal range and the azimuth of the whale at $t=t^{(s)}$ are $r^{(s)}=H_{p} E_{p}^{(s)}$ and $\theta^{(s)}$, respectively. 


$$
\begin{aligned}
& \mathcal{S}^{(1)} \equiv\left(z^{(1)}, r^{(1)}, \theta^{(1)}, v_{z}^{(1)}, v_{r}^{(1)}, \gamma_{b}^{(1)}\right), \\
& \mathcal{S}^{(s)} \equiv\left(v_{z}^{(s)}, v_{r}^{(s)}, \Delta_{\gamma}^{(s)} \mid \mathcal{S}^{(s-1)}\right) \quad \text { for } s \in\{2, \ldots, n\}
\end{aligned}
$$

given the coordinates of the first summit $\left[E^{(1)}\right.$, defined by the triplet $\left.\left(z^{(1)}, r^{(1)}, \theta^{(1)}\right)\right]$ and the whale vertical speed, horizontal speed, and change of heading in the $n$ segments. Alternatively, given the coordinates of the last summit $\left[E^{(n+1)}\right.$, defined by the triplet $\left.\left(z^{(n+1)}, r^{(n+1)}, \theta^{(n+1)}\right)\right]$ and the whale vertical speed, horizontal speed, and change of heading in the $n$ segments, each segment $\mathcal{S}^{(s)}$ is recursively defined by writing

$$
\begin{aligned}
& \mathcal{S}^{(s)} \equiv\left(v_{z}^{(s)}, v_{r}^{(s)}, \Delta_{\gamma}^{(s+1)} \mid \mathcal{S}^{(s+1)}\right) \quad \text { for } s \in\{1, \ldots, n-1\} \\
& \mathcal{S}^{(n)} \equiv\left(v_{z}^{(n)}, v_{r}^{(n)}, \gamma_{e}^{(n)}, z^{(n+1)}, r^{(n+1)}, \theta^{(n+1)}\right) .
\end{aligned}
$$

Using Eq. (3), the trajectory $\mathcal{T}$ is also entirely defined by the set of $3 n+3$ parameters

$$
\begin{aligned}
\mathcal{T} \equiv & \left(z^{(1)}, r^{(1)}, \theta^{(1)}, v_{z}^{(1)}, v_{r}^{(1)}, \gamma_{b}^{(1)},\right. \\
& \left.v_{z}^{(2)}, v_{r}^{(2)}, \Delta_{\gamma}^{(2)}, \ldots, v_{z}^{(n)}, v_{r}^{(n)}, \Delta_{r}^{(n)}\right),
\end{aligned}
$$

or alternatively, using Eq. (4), by the set of $3 n+3$ parameters

$$
\begin{aligned}
\mathcal{T} \equiv & \left(v_{z}^{(1)}, v_{r}^{(1)}, \Delta_{\gamma}^{(2)}, \ldots, v_{z}^{(n-1)},\right. \\
& \left.v_{r}^{(n-1)}, \Delta_{\gamma}^{(n)}, v_{z}^{(n)}, v_{r}^{(n)}, \gamma_{e}^{(n)}, z^{(n+1)}, r^{(n+1)}, \theta^{(n+1)}\right) .
\end{aligned}
$$

\section{B. Prior information}

By choosing $\theta^{(1)}=0, \theta^{(n+1)}$ represents the change in azimuth of the whale, relative to the research vessel, between the points $E^{(1)}$ and $E^{(n+1)}$. One can combine the definitions of $\mathcal{T}$ given in Eqs. (5) and (6) by writing

$$
\begin{aligned}
\mathcal{T} \equiv & \left(z^{(1)}, r^{(1)}, v_{z}^{(1)}, v_{r}^{(1)}, \gamma_{b}^{(1)}, v_{z}^{(2)}, v_{r}^{(2)},\right. \\
& \Delta_{\gamma}^{(2)}, \ldots, v_{z}^{(n)}, v_{r}^{(n)}, \\
& \left.\Delta_{\gamma}^{(n)}, z^{(n+1)}, r^{(n+1)}, \theta^{(n+1)}\right) .
\end{aligned}
$$

This latter definition of $\mathcal{T}$ uses $3 n+5$ parameters, that is to say redundant information. Indeed, the coordinates of the last summit $E^{(n+1)}$ can be calculated given the coordinates of the first summit $E^{(1)}$ and using the values of speeds and change of heading in the $n$ segments. The consequences of such redundancy during the estimation process will be discussed later. The aim of combining Eqs. (5) and (6) into Eq. (7) is to gather in a single definition of $\mathcal{T}$ information on the fluking and resurfacing points of the whale.

Let $z^{(A)}=0 \mathrm{~m}$ and $r^{(A)}$ be the depth and the horizontal range of the whale at the time $t=t^{(A)}$. Let $v_{z}^{(A)}$ and $v_{r}^{(A)}$ be the average vertical and horizontal speeds of the whale for $t$ $\in\left[t^{(A)}, t^{(B)}\right]$. The depth and the range of the whale at $t=t^{(1)}$ are then $z^{(1)}=v_{z}^{(A)}\left(t^{(B)}-t^{(A)}\right)$ and $r^{(1)}$. Let $z^{(D)}=0 \mathrm{~m}$ and $r^{(D)}$ be the depth and the horizontal range of the whale at the time $t=t^{(D)}$. Let $v_{z}^{(D)}$ and $v_{r}^{(D)}$ be the average vertical and horizontal speeds of the whale for $t \in\left[t^{(C)}, t^{(D)}\right]$. The depth and the range of the whale at $t=t^{(n+1)}$ are then $z^{(n+1)}=v_{z}^{(D)}\left(t^{(C)}-t^{(D)}\right)$ and $r^{(n+1)}$.

Sperm whales initiate their foraging dives by fluking-up and diving vertically (as observed from the sea surface at the very beginning of the dive). Sperm whales usually keep a constant vertical speed while descending to reach their prey and while ascending to reach the sea surface (Miller et al., 2004a). There are exceptions however: Sperm whales may, for instance, horizontally translate during the ascent likely due to the presence of conspecifics (Miller et al., 2004b). Assuming that the main objective of the whale while descending is to reach bathypelagic prey and that the main objective of the whale while ascending is to reach oxygen at the sea surface, the whale would swim vertically for $t$ $\in\left[t^{(A)}, t^{(B)}\right]$ and $t \in\left[t^{(C)}, t^{(D)}\right]$. This leads to $v_{r}^{(A)} \simeq 0 \mathrm{~m} \mathrm{~s}^{-1}$, $v_{r}^{(D)} \simeq 0 \mathrm{~m} \mathrm{~s}^{-1}, r^{(1)} \simeq r^{(A)}$, and $r^{(n+1)} \simeq r^{(D)}$. In this case $\theta^{(n+1)}$ represents the change in azimuth of the whale, relative to the research vessel, between the fluking and the resurfacing points. A visual observer can measure the parameters $z^{(1)}$, $r^{(1)}, z^{(n+1)}, r^{(n+1)}$, and $\theta^{(n+1)}$ from the research vessel. The visual measurement process is inaccurate and thus results in uncertainties on $z^{(1)}, r^{(1)}, z^{(n+1)}, r^{(n+1)}$, and $\theta^{(n+1)}$. The assumption of verticalness may also be inaccurate resulting in additional uncertainties on these variables. Such uncertainties are modeled in the following section using random variables.

\section{Likelihood}

The algorithm which will be described later is used to estimate the depth and the range of the whale during a dive, and requires the set of values of the surface echo delay of the clicks that the whale has emitted during this dive. Let $K_{e}$ be the number of echolocation clicks that the whale has emitted for $t \in\left[t^{(B)}, t^{(C)}\right]$. The author assumes that $K \leqslant K_{e}$ clicks are correctly detected (both the direct path and the surface echo). The observer then makes $K$ consistent measurements of the surface echo delay at the time $t \in\left\{t_{1}, \ldots, t_{K}\right\} \quad\left(t_{1}=t^{(B)}\right.$ and $t_{K}$ $\left.=t^{(C)}\right)$, labeled

$$
\mathcal{M}=\left(\tau\left(t_{1}\right), \ldots, \tau\left(t_{K}\right)\right) .
$$

The trajectory model $\mathcal{T}$ is close to the true trajectory that the whale follows. Let $f\left(\mathcal{T}, t_{k}\right)$ be the value at the time $t_{k}(k$ $\in\{1, \ldots, K\})$ of the surface echo delay if the whale were on the trajectory $\mathcal{T}$. Such value, however, due to the inaccuracy of the measurement and modeling processes, is not exactly equal to $\tau\left(t_{k}\right)$. The difference $\epsilon\left(\mathcal{T}, t_{k}\right)$ between the data $\tau\left(t_{k}\right)$ and the model $f\left(\mathcal{T}, t_{k}\right)$ is defined as

$$
\tau\left(t_{k}\right)=f\left(\mathcal{T}, t_{k}\right)+\epsilon\left(\mathcal{T}, t_{k}\right) \quad \text { for } k \in\{1, \ldots, K\} .
$$

The author assumes that the errors due to such inaccuracies are centered (the mean of the error is equal to zero), independent [the error made on $\tau\left(t_{i}\right)$ is independent with the error made on $\left.\tau\left(t_{j}\right), i \neq j\right]$, and of equal variance. In that case, one can model the above-described inaccuracies by interpreting them as an additive white Gaussian noise. Let $\boldsymbol{\epsilon}$ be a centered, white Gaussian noise of standard deviation $\sigma_{\tau}, \boldsymbol{\epsilon}$ $\sim \mathcal{N}\left(0, \sigma_{\tau}\right)$.

The noise on the data is modeled using the random variable $\boldsymbol{\epsilon}$. One can model the fluctuations in the values of the parameters previously defined using random variables. Let $\mathbf{E}^{(s)}, \mathbf{E}_{\mathbf{p}}^{(s)}, \mathbf{z}^{(s)}, \mathbf{r}^{(s)}$, and $\boldsymbol{\theta}^{(s)}$ be the random variables corresponding to the parameters $E^{(s)}, E_{p}^{(s)}, z^{(s)}, r^{(s)}$, and $\theta^{(s)}$ (for 
$s \in\{1, \ldots, n+1\})$. Let $\mathcal{S}^{(s)}, \mathbf{v}_{\mathbf{z}}^{(s)}, \mathbf{v}_{\mathbf{r}}^{(s)}, \gamma_{\mathbf{b}}^{(s)}, \gamma_{\mathbf{e}}^{(s)}$, and $\boldsymbol{\Delta}_{\gamma}^{(s)}$ be the random variables corresponding to the parameters $\mathcal{S}^{(s)}, v_{z}^{(s)}$, $v_{r}^{(s)}, \gamma_{b}^{(s)}, \gamma_{e}^{(s)}($ for $s \in\{1, \ldots, n\})$, and $\Delta_{\gamma}^{(s)}$ (for $s \in\{2, \ldots, n\}$ ). Let $\mathcal{T}, \mathcal{M}$, and $\boldsymbol{\tau}\left(t_{k}\right)$ be the random variables corresponding to the parameters $\mathcal{T}, \mathcal{M}$, and $\tau(k)$ (for $k \in\{1, \ldots, K\}$ ). Using such definitions, relationship (9) can be rewritten $\boldsymbol{\tau}\left(t_{k}\right)$ $=f\left(\boldsymbol{T}, t_{k}\right)+\boldsymbol{\epsilon}$. The likelihood of a single measurement $\boldsymbol{\tau}\left(t_{k}\right)$ is then

$$
p\left(\tau\left(t_{k}\right) \mid \mathcal{T}\right)=\frac{1}{\sqrt{2 \pi \sigma_{\tau}^{2}}} \exp \left[\frac{-\left[\tau\left(t_{k}\right)-f\left(\mathcal{T}, t_{k}\right)\right]^{2}}{2 \sigma_{\tau}^{2}}\right]
$$

by noting $p(x \mid y)=p(\mathbf{x}=x \mid \mathbf{y}=y)$, the value at $x$ of the conditional probability density function of the random variable $\mathbf{x}$ given the value $y$ of the random variable $\mathbf{y}$. The aforementioned assumptions on the noise $\boldsymbol{\epsilon}$ lead to the following expression of the likelihood of the measurement set $\mathcal{M}$ :

$$
p(\mathcal{M} \mid \mathcal{T})=\frac{1}{\sqrt{2 \pi \sigma_{\tau}^{2}}} \exp \left[-\frac{1}{2 \sigma_{\tau k=1}^{2}} \sum^{K}\left[\tau\left(t_{k}\right)-f\left(\mathcal{T}, t_{k}\right)\right]^{2}\right]
$$

\section{Independence between the variables}

Using the definition of the trajectory given in Eq. (7), the prior probability of a trajectory $\mathcal{T}$ is

$$
\begin{aligned}
p(\mathcal{T})= & p\left(z^{(1)}, r^{(1)}, v_{z}^{(1)}, v_{r}^{(1)}, \gamma_{b}^{(1)}, v_{z}^{(2)}, v_{r}^{(2)},\right. \\
& \left.\Delta_{\gamma}^{(2)}, \ldots, v_{z}^{(n)}, v_{r}^{(n)}, \Delta_{\gamma}^{(n)}, z^{(n+1)}, r^{(n+1)}, \theta^{(n+1)}\right),
\end{aligned}
$$

by noting $p(x)=p(\mathbf{x}=x)$, the value at $x$ of the probability density function of the random variable $\mathbf{x}$. In reality the $3 n$ +5 variables are statistically dependent, but one does not know what the dependency is. One needs to make some assumptions and simplifications with the aim of giving an analytical expression of the prior $p(\mathcal{T})$.

As mentioned earlier, the definition given in Eq. (7) is not minimal, and one can theoretically calculate $\left(\mathbf{z}^{(n+1)}, \mathbf{r}^{(n+1)}, \boldsymbol{\theta}^{(n+1)}\right)$ [related to the location of the resurfacing point] using $\left(\mathbf{z}^{(1)}, \mathbf{r}^{(1)}\right)$ [related to the location of the fluking point] and $\left(\mathbf{v}_{\mathbf{z}}^{(1)}, \mathbf{v}_{\mathbf{r}}^{(1)}, \boldsymbol{\gamma}_{\mathbf{b}}^{(1)}, \mathbf{v}_{\mathbf{z}}^{(2)}, \mathbf{v}_{\mathbf{r}}^{(2)}, \boldsymbol{\Delta}_{\boldsymbol{\gamma}}^{(2)}, \ldots, \mathbf{v}_{\mathbf{z}}^{(n)}, \mathbf{v}_{\mathbf{r}}^{(n)}, \boldsymbol{\Delta}_{\gamma}^{(n)}\right)$ [related to the underwater behavior of the whale]. The author, nevertheless, assumes from now on that the relationship among the fluking point, the resurfacing point, and the underwater behavior of the whale is too intricate to be comprehensible to an observer. In this case, there is no apparent prior relationship between $\left(\mathbf{z}^{(1)}, \mathbf{r}^{(1)}\right), \quad\left(\mathbf{v}_{\mathbf{z}}^{(1)}, \mathbf{v}_{\mathbf{r}}^{(1)}, \boldsymbol{\gamma}_{\mathbf{b}}^{(1)}, \mathbf{v}_{\mathbf{z}}^{(2)}, \mathbf{v}_{\mathbf{r}}^{(2)}, \boldsymbol{\Delta}_{\gamma}^{(2)}, \ldots, \mathbf{v}_{\mathbf{z}}^{(n)}, \mathbf{v}_{\mathbf{r}}^{(n)}, \boldsymbol{\Delta}_{\gamma}^{(n)}\right)$, and $\left(\mathbf{z}^{(n+1)}, \mathbf{r}^{(n+1)}, \boldsymbol{\theta}^{(n+1)}\right)$, that is to say that these three sets of variables are assumed to be statistically independent.

One can assume that (1) the range of the whale when fluking up, (2) the vertical speed of the whale when initiating a dive, and (3) the period of time the whale waits before clicking are statistically independent, hence the independence between $\mathbf{r}^{(1)}$ and $\mathbf{z}^{(1)}$. One can also make similar assumptions regarding the final ascent, hence the independence among $\mathbf{r}^{(n+1)}, \mathbf{z}^{(n+1)}$, and $\boldsymbol{\theta}^{(n+1)}$.

Finally, there must be a strong dependence between the parameters $\mathbf{v}_{\mathbf{z}}^{(i)}, \mathbf{v}_{\mathbf{r}}^{(i)}(i \in\{1, \ldots, n\})$, and $\left(\boldsymbol{\Delta}_{\boldsymbol{\gamma}}^{(2)}, \ldots, \boldsymbol{\Delta}_{\boldsymbol{\gamma}}^{(n)}\right)$ de- scribing the underwater behavior of the whale. Such a relationship is however unknown to an observer. One can assume that the changes in vertical speed, horizontal speed, and heading are statistically independent. One can also assume that the speed of the whale in each segment is independent of the speed of the whale in any other segment. In view of the above-presented statements, the prior probability given in Eq. (12) can be rewritten

$$
\begin{aligned}
p(\mathcal{T})= & p\left(z^{(1)}\right) p\left(r^{(1)}\right) p\left(\gamma_{b}^{(1)}\right) p\left(z^{(n+1)}\right) p\left(r^{(n+1)}\right) p\left(\theta^{(n+1)}\right) \\
& \times\left[\prod_{s=1}^{n} p\left(v_{z}^{(s)}\right) p\left(v_{r}^{(s)}\right)\right] p\left(\Delta_{\gamma}^{(2)}, \ldots, \Delta_{\gamma}^{(n)}\right) .
\end{aligned}
$$

\section{E. Probability distribution of the prior}

The author will give a more accurate description of the prior distributions appearing in Eq. (13). Using the Hammersley-Clifford theorem (Perez, 1998), choosing a joint probability density function $(\alpha>0)$

$$
p\left(\Delta_{\gamma}^{(2)}, \ldots, \Delta_{\gamma}^{(n)}\right) \propto \exp \left[-\alpha \sum_{s=2}^{n}\left|\Delta_{\gamma}^{(s)}\right|\right]
$$

ensures that $\left(\boldsymbol{\Delta}_{\boldsymbol{\gamma}}^{(2)}, \ldots, \boldsymbol{\Delta}_{\gamma}^{(n)}\right)$ is a Markov chain. By choosing such a prior, the author indicates that the underwater movement of the whale is not erratic, and that it is likely that the whale tends to swim in a given direction. The strength of this constraint (later referred to as constraint of rectilinearity) increases with $\alpha$.

One has no prior information on the heading of the whale when it starts to dive, hence $p\left(\gamma_{b}^{(1)}\right)=1 / 2 \pi$ for $\gamma_{b}^{(1)}$ $\in[-\pi, \pi]$. The prior information regarding the values of $\mathbf{r}^{(1)}$, $\mathbf{z}^{(1)},\left|\mathbf{v}_{\mathbf{z}}^{(s)}\right|, \mathbf{v}_{\mathbf{r}}^{(s)}(s \in\{1, \ldots, n\}), \mathbf{r}^{(n+1)}, \mathbf{z}^{(n+1)}$, and $\boldsymbol{\theta}^{(n+1)}$ is introduced by assigning to these random variables bell-shaped probability density functions. The probability density functions have a maximum at the most likely prior value of the parameters, and its width illustrates the confidence we grant to this most likely prior value. One could choose for instance gamma probability distributions. For practical reasons, the author has chosen truncated normal distributions. Such probability distributions are denoted $\mathcal{N}_{[a, b]}(\mu, \sigma)$ in the following $\left[(a, b) \in \overline{\mathbb{R}}^{2}, a<b, \mu \in[a, b], \sigma>0\right]$. Their probability density functions are equal to, for $x \in[a, b]$,

$$
g(x)=f(x) / \int_{a}^{b} f(t) d t
$$

with

$$
f(x)=\frac{1}{\sqrt{2 \pi \sigma^{2}}} \exp \left[-\frac{(x-\mu)^{2}}{2 \sigma^{2}}\right] .
$$

The author assumes that $\mathbf{v}_{\mathbf{z}}^{(s)}$ is positive or negative with an equal probability $1 / 2$.

\section{F. Probability distribution of the posterior}

Using Bayes' formula (Robert and Casella, 2004), the posterior of the trajectory $\mathcal{T}$ is known modulo a multiplicative constant 


$$
p(\mathcal{T} \mid \mathcal{M})=\frac{p(\mathcal{M} \mid \mathcal{T}) p(\mathcal{T})}{p(\mathcal{M})} \propto p(\mathcal{M} \mid \mathcal{T}) p(\mathcal{T})
$$

Using Eqs. (11) and (13), one can calculate the posterior $p(\mathcal{T} \mid \mathcal{M})$ of the trajectory $\mathcal{T}$ modulo a multiplicative constant. The mean $\hat{\mathcal{T}}$ of the posterior $p(\mathcal{T} \mid \mathcal{M})$ is the trajectory which best fits the data $\mathcal{M}$ in the minimal mean square error (MMSE) sense (Robert and Casella, 2004).

\section{G. MCMC algorithm}

The analytical expression of the probability $p(\mathcal{T} \mid \mathcal{M})$ is known modulo a multiplicative constant. One cannot directly draw samples using Eqs. (11), (13), and (16). However, one can use a Markov Chain Monte Carlo (MCMC) algorithm to draw samples of the posterior. Such an algorithm can generate samples $\mathcal{T}[i]\left(i \in\left\{I_{1}, \ldots, I_{2}\right\},\left(I_{1}, I_{2}\right) \in \mathbb{N}^{2}, I_{2}>I_{1}\right.$ distributed according to the posterior $p(\mathcal{T} \mid \mathcal{M})$. The mean of such samples

$$
\hat{\mathcal{T}}=\frac{1}{I_{2}-I_{1}+1} \sum_{i=I_{1}}^{I_{2}} \mathcal{T}[i]
$$

is then an estimate of the optimal trajectory $\hat{\mathcal{T}}$ in the MMSE sense. One can use the Metropolis-Hastings algorithm (Robert and Casella, 2004) to draw such samples. Choose an initial value $\mathcal{T}[0]$ of the trajectory, and, at iteration $i(i$ $\left.\in\left\{1, \ldots, I_{2}\right\}\right)$, draw a trajectory sample $\mathcal{T}_{d}[i]$. Calculate the acceptance ratio of this sample to determine if the new sample $\mathcal{T}_{d}[i]$ is accepted $\left(\mathcal{T}[i] \equiv \mathcal{T}_{d}[i]\right)$ or rejected $(\mathcal{T}[i]$ $\equiv \mathcal{T}[i-1]$ ) [see Robert and Casella (2004) for a description on how to draw samples and how to calculate acceptance ratios]. The algorithm converges after $I_{1}$ iterations, and samples drawn for $i \geqslant I_{1}$ will be distributed according to $p(\mathcal{T} \mid \mathcal{M})$.

To accelerate convergence, one can do Gibbs sampling. Instead of drawing a whole new trajectory at each iteration $i$, draw the summits one by one. For $i \in\left\{1, \ldots, I_{2}\right\}$ and for $s$ $\in\{1, \ldots, n+1\}$, draw a trajectory sample $\mathcal{T}_{d}^{(s)}[i]$ of $\mathcal{T}$. Calculate the acceptance ratio of this sample to determine if it is accepted $\left(\mathcal{T}^{(s)}[i] \equiv \mathcal{T}_{d}^{(s)}[i]\right)$ or rejected [see Robert and Casella (2004) for a description of the Metropolis-Hastings algorithm using Gibbs sampling and try the software SBPLASH to see how the algorithm is implemented by the author]. The estimate of the trajectory is

$$
\hat{\mathcal{T}}=\frac{1}{(n+1)\left(I_{2}-I_{1}+1\right)} \sum_{i=I_{1}}^{I_{2}} \sum_{s=1}^{n+1} \mathcal{T}^{(s)}[i] .
$$

Instead of using samples for $i \in\left\{I_{1}, \ldots, I_{2}\right\}$ of a single large Markov chain to calculate $\hat{\mathcal{T}}$, one can use samples for $i \in\left\{I_{1}, \ldots, I_{2}^{\prime}\right\}$ of $C$ smaller subchains, with $I_{2}^{\prime}=I_{1}+\Delta_{I}^{\prime}, \Delta_{I}^{\prime}$ $=\Delta_{I} / C, \Delta_{I}=I_{2}-I_{1}$. In theory, if convergence is reached (that is to say $I_{1}$ is large enough), each method will provide the same result. In practice, the second method requires a lower total amount of samples, as it requires less than $\Delta_{I} / C$ samples per subchain to lead to a correct estimate $\hat{\mathcal{T}}$. The author has used the subchain method. In this case, by label- ing $\mathcal{T}^{(s)}[i][c]$ the trajectory samples $(c \in\{1, \ldots, C\}, i$ $\left.\in\left\{1, \ldots, I_{2}\right\}, s \in\{1, \ldots, n+1\}\right)$, the final estimate will be

$$
\hat{\mathcal{T}}=\frac{1}{C(n+1)\left(I_{2}^{\prime}-I_{1}+1\right)} \sum_{c=1}^{C} \sum_{i=I_{1}}^{I_{2}^{\prime}} \sum_{s=1}^{n+1} \mathcal{T}^{(s)}[i][c] .
$$

\section{H. Data set}

The author has run simulations using the free software SBPLASH implemented in MATLAB. Source and data files are available at http://www.ensat.fr/pers/laplanche/sbplash. The author did not have complete data (i.e., both trajectory and acoustic data) gathered during field experiments to test the algorithm. The efficiency of the algorithm is illustrated using synthetic data. The following trajectory was used in the simulations.

The whale flukes up and vertically dives at $t^{(A)}=0 \mathrm{~min}$, at a speed $v_{z}^{(A)}=1 \mathrm{~m} \mathrm{~s}^{-1}$, and at a range $r^{(A)}=500 \mathrm{~m}$ west of the hydrophone. The whale starts clicking at $t^{(B)}=0.5 \mathrm{~min}$. The descent lasts $10 \mathrm{~min}$. At $t=10 \mathrm{~min}$, the whale starts a $10 \mathrm{~min}$ swim, north at $v_{r}=1 \mathrm{~m} \mathrm{~s}^{-1}$ and upwards at $v_{z}=-0.5 \mathrm{~m} \mathrm{~s}^{-1}$. At $t=20 \mathrm{~min}$, the whale starts a $10 \mathrm{~min}$ swim, east at $v_{r}=1 \mathrm{~m} \mathrm{~s}^{-1}$ and downwards at $v_{z}=0.5 \mathrm{~m} \mathrm{~s}^{-1}$. At $t^{(C)}=30 \mathrm{~min}$, the whale stops clicking and starts a $10 \mathrm{~min}$ vertical ascent to the sea surface at $v_{z}=-1 \mathrm{~m} \mathrm{~s}^{-1}$. The whale resurfaces at $t^{(D)}=40 \mathrm{~min}$. The depth, range, vertical speed, and horizontal speed of the whale when following this trajectory are given in Figs. 3-6, respectively.

The acoustic activity of the whale is modeled by assuming that it emitted $K=1770$ clicks for $t \in\left[t^{(B)}, t^{(C)}\right]$ at $1 \mathrm{click} / \mathrm{s}$ with no interruption. The hydrophone is at a $70 \mathrm{~m}$ depth and the speed of sound is $c=1500 \mathrm{~m} \mathrm{~s}^{-1}$. Every click that the whale emitted is assumed to have been detected (both direct path and surface reflection) leading to $K$ measurements of the surface echo delay $\tau$. The measurement process of the delays is not free from errors (Wahlberg et al., 2001). A centered white Gaussian noise of standard deviation $\sigma_{\tau}=1 \mathrm{~ms}$ has been added to the $\tau$ data to simulate such inaccuracy. The noisy values of $\tau$ compose the data set denoted earlier as $\mathcal{M}$. Such values are plotted in Fig. 7.

The above-described trajectory is unknown to the observer. He knows however the data set $\mathcal{M}$ and has some visual information on the fluking-up and resurfacing points. The range of the whale when fluking-up is measured as $r^{(A)}=500 \pm 25 \mathrm{~m}\left[\mathbf{r}^{(A)} \sim \mathcal{N}_{[0,+\infty]}(500,25)\right]$. The vertical speed of the whale for $t \in\left[t^{(A)}, t^{(B)}\right]$ (when initiating a dive) is assumed to be equal to $v_{z}^{(A)}=1 \pm 0.1 \mathrm{~m} \mathrm{~s}^{-1}\left[\mathbf{v}_{\mathbf{z}}^{(A)} \sim \mathcal{N}_{[0,+\infty]}\right.$ $(1,0.1)]$. The depth of the whale when it starts clicking is then $z^{(B)}=30 \pm 3 \mathrm{~m}\left[\mathbf{z}^{(B)} \sim \mathcal{N}_{[0,+\infty]}(30,3)\right]$. The prior vertical and horizontal speeds of the whale for $t \in\left[t^{(B)}, t^{(C)}\right]$ follow the same truncated normal probability distribution $\mathcal{N}_{[0,+\infty]}$ $(0,0.75)$. The whale resurfaces at a range $r^{(D)}=630 \pm 25 \mathrm{~m}$ $\left[\mathbf{r}^{(D)} \sim \mathcal{N}_{[0,+\infty]}(630,25)\right]$. The vertical speed of the whale for $t \in\left[t^{(C)}, t^{(D)}\right]$ (when ending a dive) is assumed to be equal to $v_{z}^{(D)}=-1 \pm 0.1 \mathrm{~m} \cdot \mathrm{s}^{-1} \quad\left[\left|\mathbf{v}_{\mathbf{z}}^{(A)}\right| \sim \mathcal{N}_{[0,+\infty]}(1,0.1)\right]$. The whale stops clicking at a depth $z^{(C)}=600 \pm 60 \mathrm{~m}\left[\mathbf{z}^{(C)} \sim \mathcal{N}_{[0,+\infty]}\right.$ $\times(600,60)]$. The change of azimuth of the whale between 

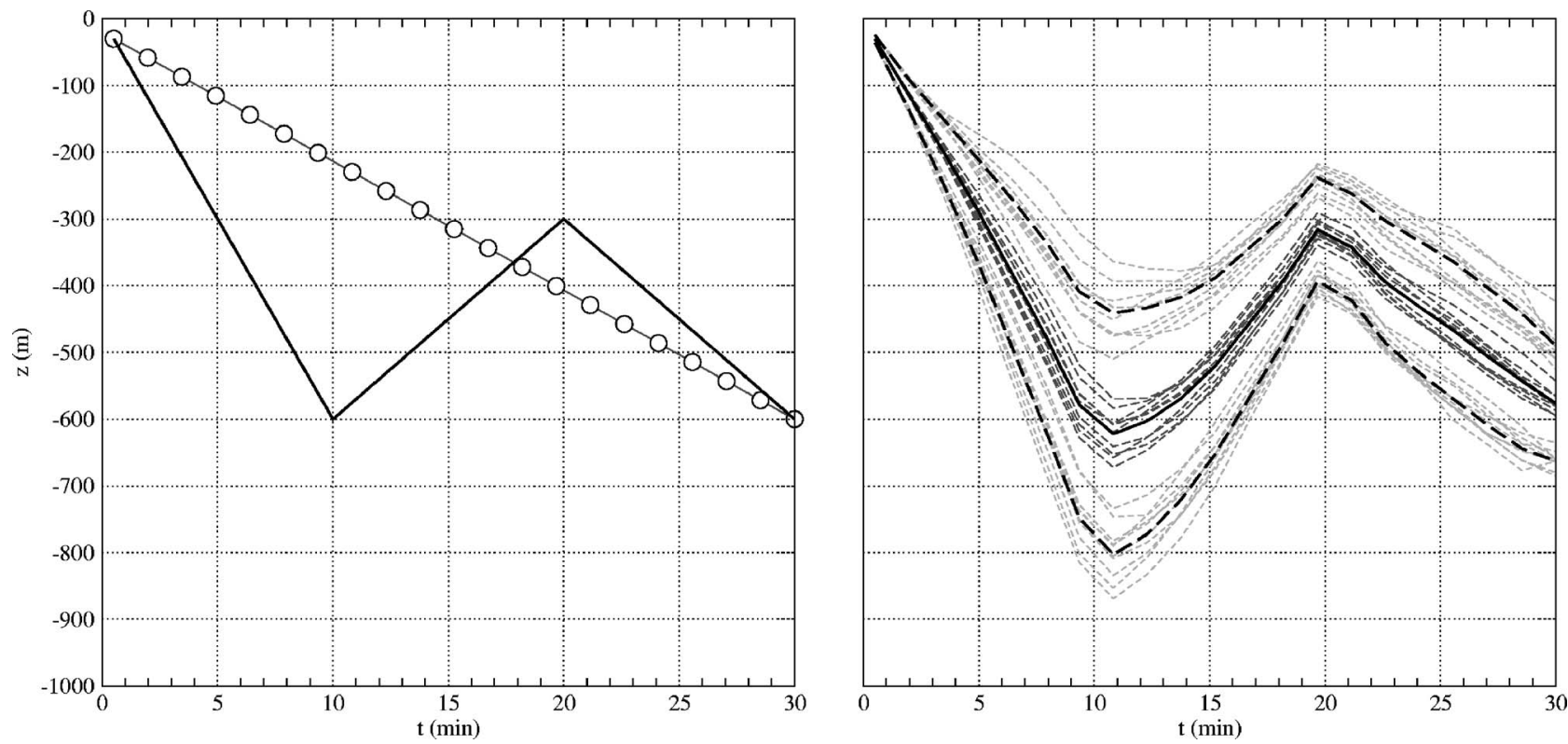

FIG. 3. True value (left, thick solid line) and initial value used in the MCMC algorithm (left, circles) of the depth $z$ of the sperm whale throughout the dive. Estimation of the depth of the sperm whale (right, thick line) plus/minus twice its standard deviation (right, thick dashed line) for $t \in\left[t^{(B)}, t^{(C)}\right]$ using the data set presented in Fig. 7 and the ten 21 000-iteration Markov subchains. Partial estimations of the depth of the sperm whale (right, thin dark dashed lines) plus/minus twice their standard deviation (right, thin bright dashed lines) for each 21,000-iteration Markov subchain.

the fluking-up and resurfacing points is $\theta^{(n+1)}=\theta^{(D)}$ $=71.5 \pm 1^{\circ}\left[\boldsymbol{\theta}^{(n+1)} \sim \mathcal{N}_{[0,360]}(71.5,1)\right]$. The choice of these priors is discussed later.

A MCMC algorithm theoretically converges no matter what initial value is chosen (Robert and Casella, 2004). The initial value of the trajectory is in the author's simulations the rectilinear, constant speed trajectory linking the prior locations of the points $E^{(1)}$ and $E^{(n+1)}$ (Figs. 3-6). The corresponding values of $\tau$ are given in Fig. 7. The trajectory is decomposed into $n=20$ segments. The parameters $\left(\alpha, \sigma_{\tau}\right.$, and the parameters of the truncated normal distributions) could be estimated while estimating the trajectory parameters
(Robert and Casella, 2004). These parameters here are constants which are empirically chosen. The author has chosen $\alpha=2$ and $\sigma_{\tau}=10 \mathrm{~ms}$. Such a choice is discussed later.

\section{RESULTS}

\section{A. Convergence}

In this example, the algorithm generates trajectories with $\tau$ values close to the data after $I_{0}=100$ iterations. Samples are considered after $I_{1}=1000$ iterations. The following results required the use of $C=10$ Markov subchains, $I_{2}^{\prime}$ $=21000$ iterations per chain $\left(\Delta_{I}=200000\right)$. The acceptance
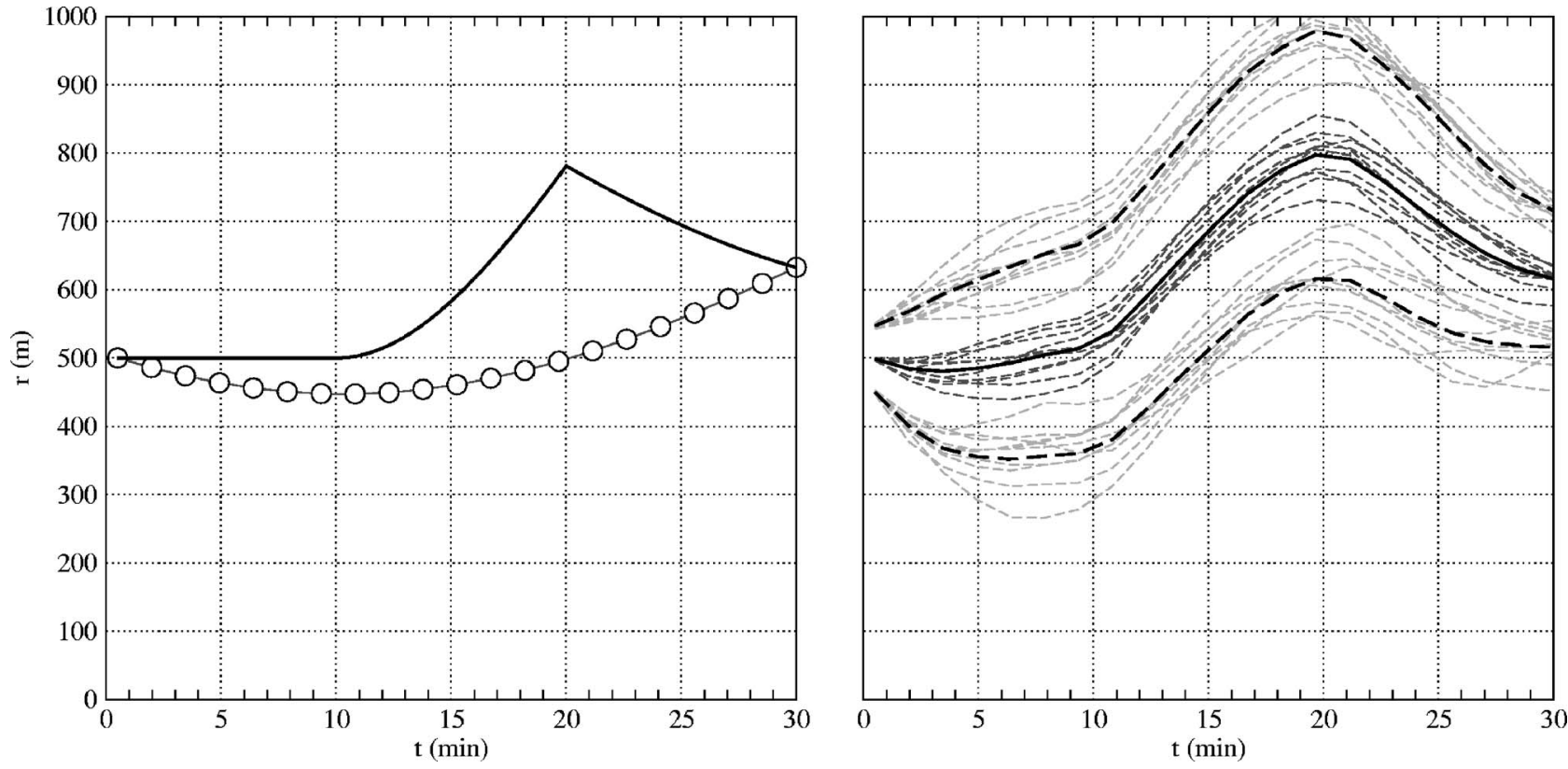

FIG. 4. True, initial, and estimated values of the range $r$ of the sperm whale throughout the dive. See Fig. 3 for detailed comments. 

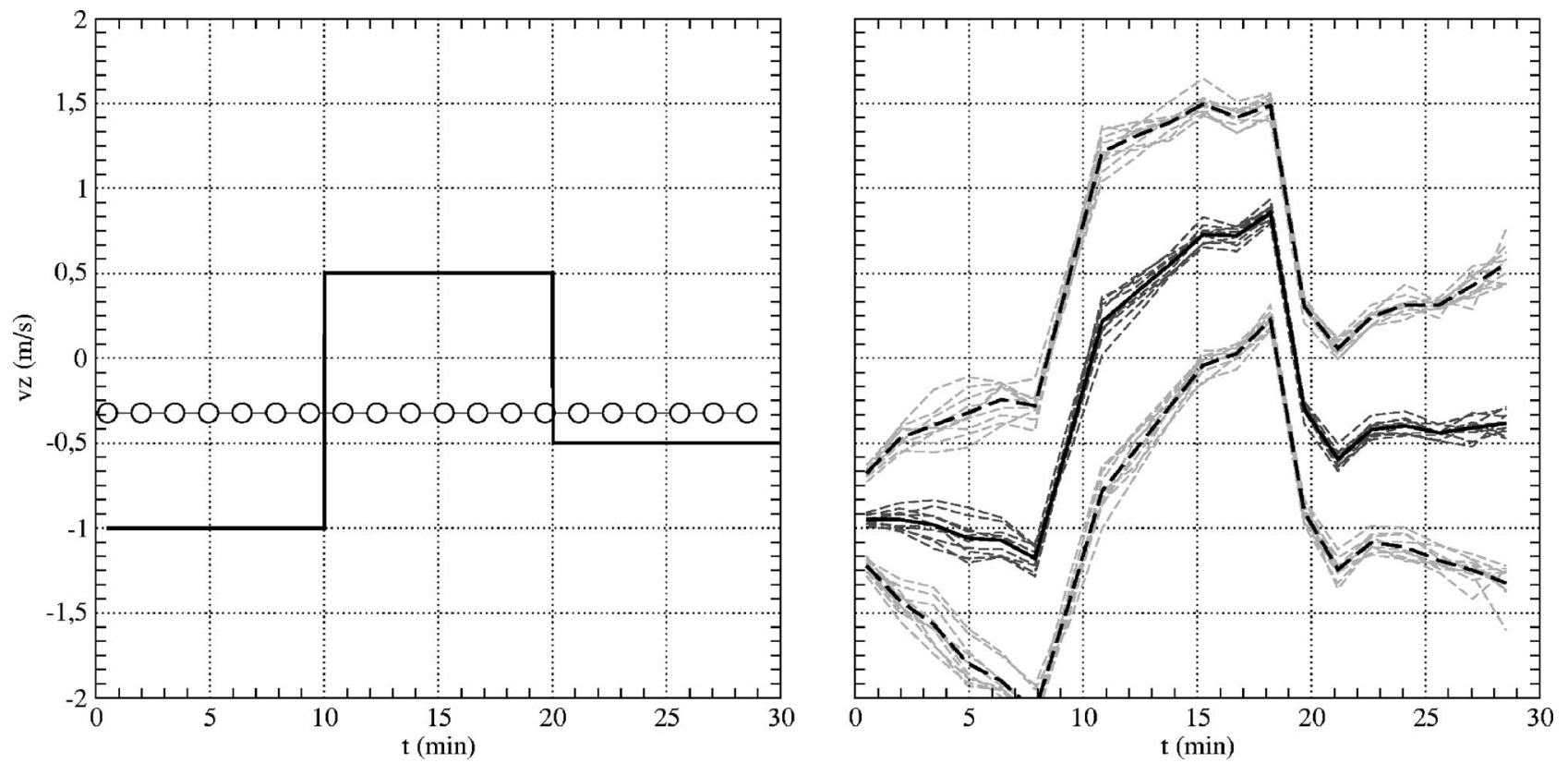

FIG. 5. True, initial, and estimated values of the vertical speed $v_{z}$ of the sperm whale throughout the dive. See Fig. 3 for detailed comments.

rate is higher for $i<I_{0}$ and stabilizes for $i \geqslant I_{0}$. In the present test, the algorithm accepted 140 (standard deviation: 21.5) new summit every 100 iterations for $i \geqslant I_{0}$. Draws of the first $(s=1)$ and last $(s=n+1)$ summits are more often accepted than the others $(2 \leqslant s \leqslant n)$, given the different constraints and priors they are bound to.

\section{B. Depth, range, and speeds}

The vertical and horizontal speeds of the whale are estimated using Eq. (19). Results are given in Figs. 5 and 6. The estimate of the depth and the range of the whale are calculated using these values. Results are given in Fig. 3 and 4. Results of $v_{z}, v_{r}, z$, and $r$ are plotted plus/minus twice their

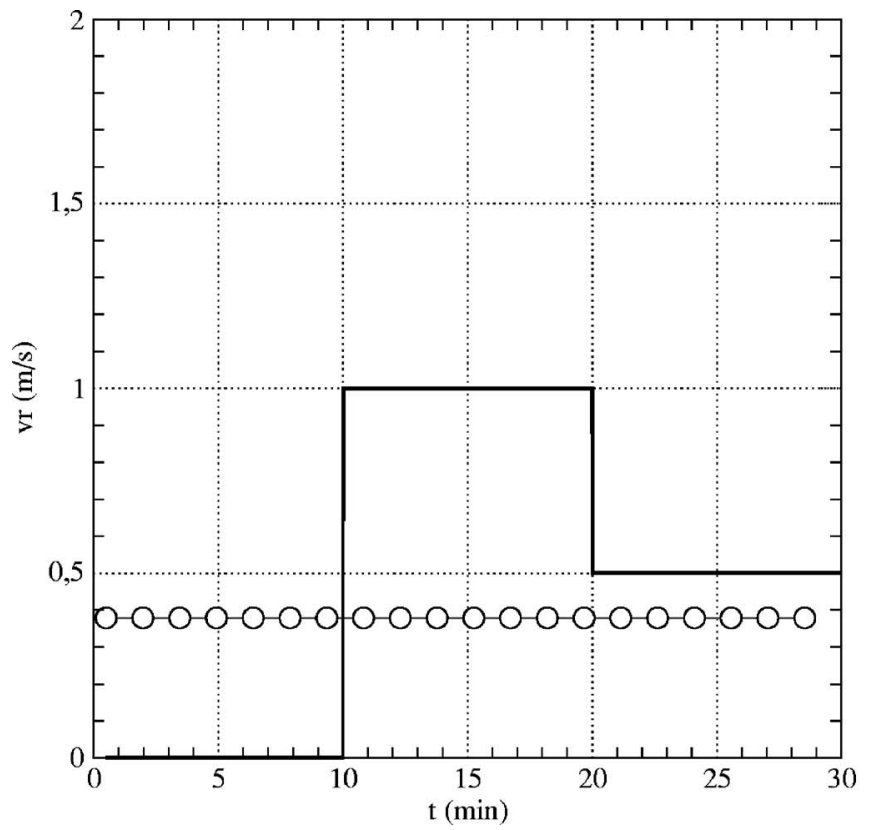

standard deviation. The standard deviation $\sigma_{\mathcal{T}}$ of the posterior $p(\mathcal{T} \mid \mathcal{M})$ is also estimated using the trajectory samples by writing

$$
\hat{\sigma}_{\mathcal{T}}^{2}=\frac{1}{C(n+1)\left(I_{2}^{\prime}-I_{1}+1\right)} \sum_{c=1}^{C} \sum_{i=I_{1}}^{I_{2}^{\prime}} \sum_{s=1}^{n+1}\left(\mathcal{T}^{(s)}[i][c]-\hat{\mathcal{T}}\right)^{2} .
$$

Although such an estimator does not take into account any correlation existing between parameters or trajectory samples.

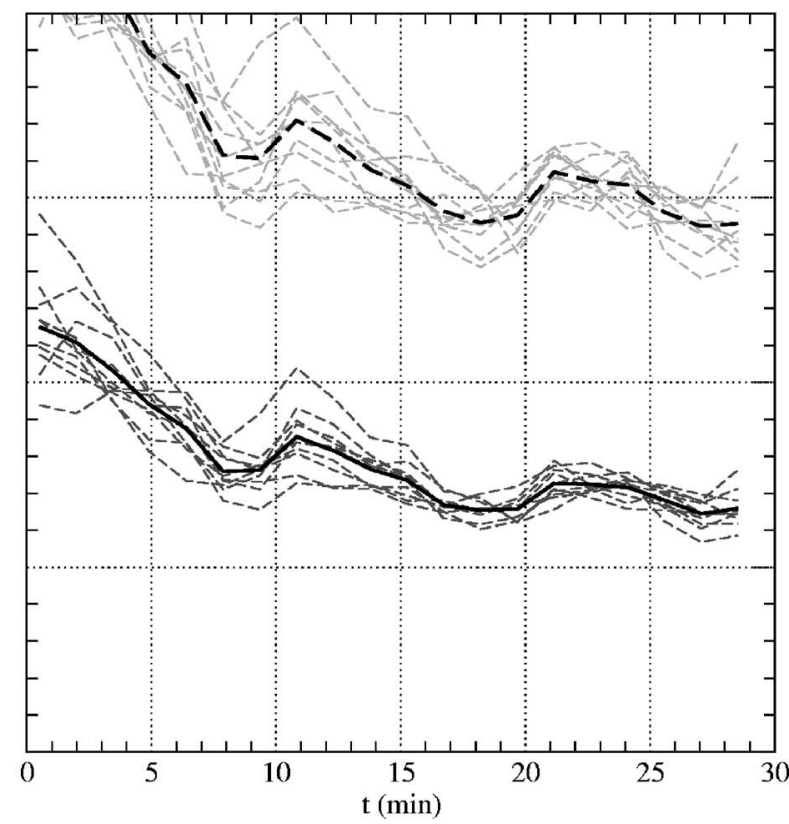

FIG. 6. True, initial, and estimated values of the horizontal speed $v_{r}$ of the sperm whale throughout the dive. See Fig. 3 for detailed comments. 


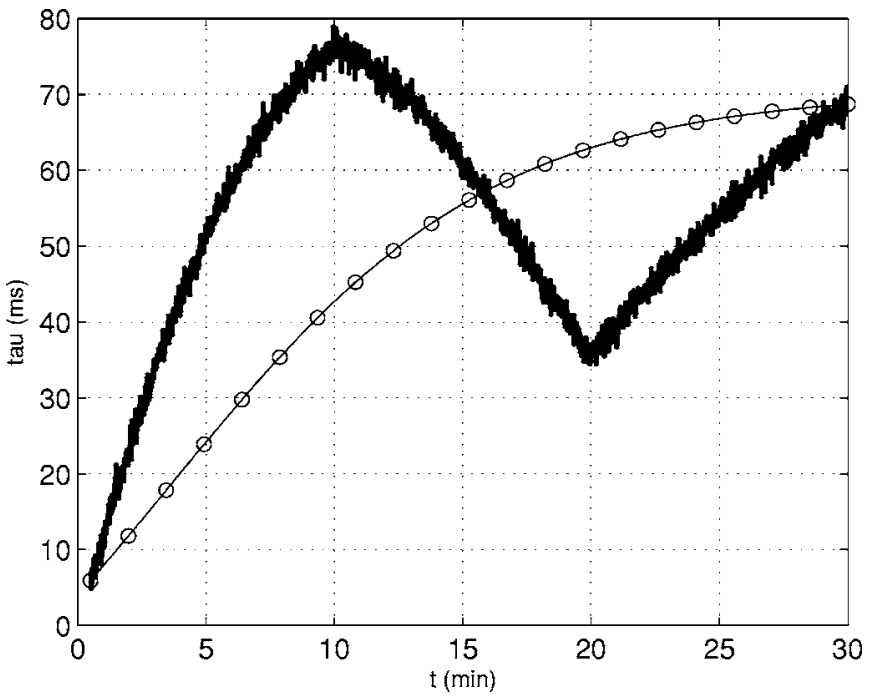

FIG. 7. Surface echo delay $\tau$ of the $K=1771$ clicks emitted by the sperm whale during the dive depicted in Figs. 3-6. The solid line describes the data set $\mathcal{M}$ used in the tests. The circles represent the $\tau$ values of the trajectory used to initiate the algorithm.

\section{DISCUSSION AND CONCLUSION}

The algorithm correctly estimates the depth and the range of the whale throughout the dive (Figs. 3 and 4). The estimates $\hat{z}$ and $\hat{r}$ differ on average from the true values by 24 and $34 \mathrm{~m}$, respectively. The application of this method shows that, indeed, useful information on the depth and the range of the phonating sperm whale is contained in the values of the surface echo delays, and that a proper algorithm can obtain such information. The author has, however, tested the method using only synthetic data. The use of a four-piece trajectory, noisy delay data, uncertain visual cues and priors, and the quality of the results made this first test very promising. Nevertheless, confronting the algorithm with real data, for instance data gathered using far-field acoustic recordings plus digital tagging (Zimmer et al., 2003), is still required for validation.

The quality of the estimation of $v_{r}$ is however arguable (Figs. 5 and 6). One should find $v_{z}=1 \mathrm{~m} \mathrm{~s}^{-1}$ and $v_{r}$ $=0 \mathrm{~m} \mathrm{~s}^{-1}$ for $t \in[0.5,10], v_{z}=-0.5 \mathrm{~m} \mathrm{~s}^{-1}$ and $v_{r}=1 \mathrm{~m} \mathrm{~s}^{-1}$ for $t \in] 10,20]$, and $v_{z}=0.5 \mathrm{~m} \mathrm{~s}^{-1}$ and $v_{r}=1 \mathrm{~m} \mathrm{~s}^{-1}$ for $t$ $\in] 20,30]$. The estimate of $v_{z}$ is correct, because the absolute vertical speed of the whale $\left(v_{z}^{(\mathrm{abs})}\right.$, in the terrestrial reference frame) is equal to the apparent vertical speed of the whale $\left(v_{z}^{\text {(app) }}\right.$, from the hydrophone point of view). The estimate of $v_{r}$ is wrong, however, because the absolute horizontal speed of the whale $\left(v_{r}^{(\mathrm{abs})}\right)$ is not equal to the apparent radial horizontal speed of the whale $\left(v_{r}^{(\mathrm{app})}\right)$. One can gather information, using a single hydrophone, on $v_{z}^{(\text {app })}$ and $v_{r}^{(\text {app })}$. It is $v_{z}$ $\equiv v_{z}^{(\mathrm{abs})}$ and $v_{r} \equiv v_{r}^{(\mathrm{abs})}$ however that are used in the trajectory model presented in Eq. (7). The estimate of $v_{r}^{(\mathrm{abs})}$ is wrong simply because $v_{r}^{(\mathrm{abs})}$ cannot be estimated using the $\tau$ values. Correct values of $v_{z}^{(\mathrm{abs})}$ and $v_{r}^{\text {(abs) }}$ could be used to reconstruct the trajectory of the whale in three dimensions. It does not seem possible, without making stronger and speculative hypotheses regarding the behavior of the whale (Laplanche et al., 2005), or without using additional information (Tiemann et al., 2006), to reconstruct a three-dimensional trajectory using a single hydrophone.

This Bayesian method has some weaknesses. The algorithm requires, to start, the whole set of values of the surface echo delay and the measurement of the location of the resurfacing point. Monte Carlo sampling is also very time consuming. The algorithm requires more time using a higher number $(n)$ of segments $(3 n+5$ parameters to estimate) and a higher number of iterations $\Delta_{I}$. For such reasons, the algorithm cannot be applied in real-time. Monte Carlo sampling could however be replaced by a faster optimization algorithm of comparable effectiveness. Kalmann filtering has been extensively used as a trajectory estimator in other fields of science. It could be interesting to use this method to filter the $\mathcal{M}$ data set to reconstruct the trajectory of the whale.

The algorithm converges, no matter what initial value of the trajectory and what instrumental probability distributions are chosen (Robert and Casella, 2004). There is not, however, a strong theory defining when convergence is reached. The choice of $I_{1}$ and $I_{2}^{\prime}$ is empirical in this example. The author stopped the simulations (that is to say $I_{2}^{\prime}$ had been chosen) when the prior probability and the likelihood of the samples $\mathcal{T}^{(s)}[i][c](c \in\{1, \ldots, C\}, s \in\{1, \ldots, n+1\}, i$ $\left.\in\left\{1, \ldots, I_{2}^{\prime}\right\}\right)$ moved around a stable mean for $\Delta_{I}^{\prime}=20000$ iterations. Samples for $i \in\left\{I_{1}, \ldots, I_{2}^{\prime}\right\}$ with $I_{1}=I_{2}^{\prime}-\Delta_{I}^{\prime}$ were considered in the estimation of the mean and the standard deviation of the posterior. It could be interesting to use a convergence diagnosis, like the criterion proposed by Gelman and Rubin (1992), to make a more rigorous choice of $I_{1}$ and $I_{2}^{\prime}$. The author is, however, confident regarding the choice of $I_{1}$ for the given data set. A margin $\left(I_{1}>I_{0}\right)$ has been considered to make the choice of $I_{1}$ correct for different values of the parameters of the priors. The choice of $I_{2}^{\prime}$ is much more difficult to make. If $I_{1}$ is correctly chosen, the samples $\mathcal{T}^{(s)}[i][c]\left(i \geqslant I_{1}\right)$ are drawn according to the posterior of $\mathcal{T}$. But a given amount of samples $\hat{\Delta}_{I}$ is required to correctly sample the posterior. The use of $\Delta_{I} \ll \hat{\Delta}_{I}$ samples in Eq. (19) and (20) might lead to inaccurate estimates. Such a phenomenon is illustrated in Figs. 3 and 4. The use of $\Delta_{I}^{\prime}$ $=20000$ samples is not enough to correctly estimate $\hat{\mathcal{T}}$. The use of $\Delta_{I}=200000$ samples leads to a correct estimate.

Assuming that the chosen set of samples correctly represents the posterior, one can, by applying Eq. (19), find $\hat{\mathcal{T}}$. The value $\hat{\mathcal{T}}$ describes the set of parameters which best fits the data in the MMSE sense. Depending on the shape of the posterior, there may be, however, differences between $\hat{\mathcal{T}}$ and the true value. This is a shortcoming inherent to Bayesian estimation techniques and this will not be discussed any further. Additional work is required to evaluate this issue regarding the method proposed by the author.

The parameters used in the probability distributions of the priors have been fixed and empirically chosen. The parameters $(\mu, \sigma)$ of the prior truncated normal distributions of $\mathbf{z}^{(1)}, \mathbf{r}^{(1)}, \mathbf{z}^{(n+1)}, \mathbf{r}^{(n+1)}$, and $\boldsymbol{\theta}^{(n+1)}$ have been chosen such that they represent, in the most realistic way, the prior information that one has on the values of these parameters. The standard deviations of such priors have been chosen great 
enough so that the choice of their mean is not critical. If the assumption of verticalness of the beginning of the descent and the ascent proves to be inexact, larger standard deviations of these priors should be considered. Using information, accurate or not, on the fluking and resurfacing points is however very helpful. The author did not manage to estimate the depth and the range of the whale or the location of the resurfacing point using only the echo delay data and the constraint of rectilinearity.

The prior vertical and horizontal speeds of the whale for $t \in\left[t^{(B)}, t^{(C)}\right]$ follow the same truncated normal distribution $\mathcal{N}_{[0,+\infty]}(0,0.75)$. The aim of this prior is to bring the information that it is highly unlikely that the speed of the whale is greater than a few $\mathrm{m} \mathrm{s}^{-1}$. Choosing a standard deviation of $0.5 \mathrm{~m} \mathrm{~s}^{-1}$ would not be realistic as it would lead to a small $(5 \%)$ chance of speed greater than $1 \mathrm{~m} \mathrm{~s}^{-1}$. Choosing a standard deviation of $1 \mathrm{~m} \mathrm{~s}^{-1}$ would not be reasonable either as it would lead to a small but not negligible $(5 \%)$ chance of speed greater than $2 \mathrm{~m} \mathrm{~s}^{-1}$. The mean choice of standard deviation $\left(0.75 \mathrm{~m} \mathrm{~s}^{-1}\right)$ seems reasonable. This choice is not critical since values close to this mean lead to consistent results. However, choosing a too low $\left(0.5 \mathrm{~m} \mathrm{~s}^{-1}\right)$ or a too high $\left(1 \mathrm{~m} \mathrm{~s}^{-1}\right)$ standard deviation of the prior speed leads to inaccurate estimates of the depth and the range of the whale throughout the dive. As mentioned previously, one can sample and estimate this standard deviation while sampling and estimating the trajectory parameters. An optimal value of the standard deviation of the prior speed could be found this way.

The correct choice of $\sigma_{\tau}$ and $\alpha$ is important. The acceptance ratio is calculated using the posterior probability (Robert and Casella, 2004). The posterior probability is a function of the likelihood and the prior probability $p\left(\Delta_{\gamma}^{(2)}, \ldots, \Delta_{\gamma}^{(n)}\right)$ [Eq. (16)]. Both terms are exponentials modulo a multiplicative constant. The natural logarithm of the posterior is

$$
\begin{aligned}
\log [p(\mathcal{T} \mid \mathcal{M})]= & -\frac{1}{2 \sigma_{\tau}^{2}} \sum_{k=1}^{K}\left[\tau\left(t_{k}\right)-f\left(\mathcal{T}, t_{k}\right)\right]^{2}-\alpha \sum_{s=2}^{n}\left|\Delta_{\gamma}^{(2)}\right| \\
& +\cdots
\end{aligned}
$$

The parameters $\sigma_{\tau}$ and $\alpha$ determine the relative importance (1) of the matching of the data $\mathcal{M}$ and (2) of the constraint of rectilinearity, respectively. The smaller the value of $\sigma_{\tau}$ the greater the importance of the data. The larger the value of $\alpha$ the greater the importance of the constraint of rectilinearity. The value of $\sigma_{\tau}$ can be sensitively chosen to representing the similarity of the trajectory model to the truth. The choice of $\alpha$ is purely empirical however. The author here has chosen a value $\alpha=2$ which makes the importance of the constraint of rectilinearity relative to the constraint of the data (1) negligible for $i \in\left\{1, \ldots, I_{0}\right\}$ and (2) comparable for $i$ $\in\left\{I_{1}, \ldots, I_{2}^{\prime}\right\}$. This ensures that (1) every trajectory sample fitting to the data (rectilinear or not) may be accepted when starting the simulation, and that (2) trajectories fitting to the data and rectilinear are more likely to be accepted than trajectories fitting to the data but less rectilinear. In the simulations, a lower value of $\alpha(\alpha \leqslant 1)$ made the constraint of rectilinearity negligible even after convergence. A large value of $\alpha(\alpha \geqslant 5)$ made the constraint of rectilinearity too strong, and did not lead to a sensitive sampling of the posterior. Such statements are still empirical and qualitative, and more work is required to evaluate this issue. The value of $\alpha$ may also vary between sperm whales and dives depending on the click rate. As stated regarding the standard deviation of the prior speed, an optimal value of $\alpha$ could be estimated while sampling and estimating the trajectory parameters.

Nevertheless, having stated the weak points, the method also has many advantages. These first results are very encouraging. Not requiring seafloor echoes, the method could be used to estimate the depth and the range of foraging sperm whales using a single hydrophone in any location no matter what the depth, the relief, or the constitution of the seafloor might be. It could also be interesting to build a similar trajectory model and apply a Bayesian method and a Monte Carlo algorithm to combine TOAD data measured using an array of two (Thode, 2004) or more hydrophones.

\section{ACKNOWLEDGMENTS}

An earlier version of this work has been presented at the 150th meeting of the ASA. The author could not have participated in this meeting without the financial help of the Association Dirac. Such participation also greatly encouraged the author to carry out this project. The author thanks A. Alibert for her input on the abstract, both reviewers for the examination of the manuscript, and J. Y. Tourneret for providing constructive comments. The author is also very grateful to $\mathrm{M}$. Lonergan for reviewing an earlier version of the manuscript and K. Hunter, R. Allsopp, and M. Adam for refining the English.

Backus, R., and Schevill, W. (1966). "Physeter clicks," in Whales, Dolphins and Porpoises, edited by K. Norris (University of California Press, Berkeley), pp. 510-527.

Gelman, A., and Rubin, D. (1992). "Inference from iterative simulation using multiple sequences," Stat. Sci. 7, 457-511.

Laplanche, C., Adam, O., Lopatka, M., and Motsch, J. (2005). "Male sperm whale acoustic behavior observed from multipaths at a single hydrophone," J. Acoust. Soc. Am. 118, 2677-2687.

Miller, P., Johnson, M., and Tyack, P. (2004a). "Sperm whale behaviour indicates the use of echolocation click buzzes 'creaks' in prey capture," Proc. R. Soc. London, Ser. B 271, 2239-2247.

Miller, P., Johnson, M., Tyack, P., and Terray, E. (2004b). "Swimming gaits, passive drag and buoyancy of diving sperm whales Physeter macrocephalus," J. Exp. Biol. 207, 1953-1967.

Møhl, B., Wahlberg, M., Madsen, P., Heerfordt, A., and Lund, A. (2003). "The monopulsed nature of sperm whale clicks," J. Acoust. Soc. Am. 114, 1143-1154.

Møhl, B., Wahlberg, M., Madsen, P., Miller, L., and Surlykke, A. (2000). "Sperm whale clicks: Directionality and source level revisited," J. Acoust. Soc. Am. 107, 638-648.

Perez, P. (1998). "Markov random fields and images," CWI Q. 11-4, 413437.

Robert, C., and Casella, G. (2004). Monte Carlo Statistical Methods, 2nd ed. (Springer, New York).

Spiesberger, J. (2001). "Hyperbolic location errors due to insufficient numbers of receivers," J. Acoust. Soc. Am. 109, 3076-3079.

Spiesberger, J. (2005). "Probability distributions for locations of calling animals, receivers, sound speeds, winds, and data from travel time differences," J. Acoust. Soc. Am. 118, 1790-1800.

Thode, A. (2004). "Tracking sperm whale (Physeter macrocephalus) dive profiles using a towed passive acoustic array," J. Acoust. Soc. Am. 116, 245-253.

Thode, A., Mellinger, D., and Stienessen, S. (2002). "Depth-dependent acoustic features of diving sperm whales (Physeter macrocephalus) in the Gulf of Mexico," J. Acoust. Soc. Am. 112, 308-321. 
Tiemann, C., Thode, A., Straley, J., O'Connell, V., and Folkert, K. (2006) "Three-dimensional localization of sperm whales using a single hydrophone," J. Acoust. Soc. Am. 120, 2355-2365.

Urick, R. (1983). Principles of Underwater Sound, 3rd ed. (McGraw-Hill, New York).

Villadsgaard, A., Wahlberg, M., and Tougaard, J. (2007). "Echolocation signals of wild harbour porpoises, Phocoena phocoena" J. Exp. Biol. 210, $56-64$.

Wahlberg, M., Møhl, B., and Madsen, P. (2001). "Estimating source position accuracy of a large-aperture hydrophone array for bioacoustics," J. Acoust.
Soc. Am. 109, 397-406.

Watkins, W., and Schevill, W. (1972). "Sound source location with a threedimensional hydrophone array," Deep-Sea Res. 19, 691-706.

Whitehead, H., and Weilgart, L. (1990). "Click rates from sperm whales," J. Acoust. Soc. Am. 87, 1798-1806.

Zimmer, W., Johnson, M., D'Amico, A., and Tyack, P. (2003). “Combining data from a multisensor tag and passive sonar to determine the diving behavior of a sperm whale (Physeter macrocephalus)," IEEE J. Ocean. Eng. 28-1, 13-28. 